besseres progressionsfreies Überleben und zumindest kein schlechteres Gesamtüberleben unter Pembrolizumab als unter Ipilimumab. Somit könnte die PD-L1-Positivität zwar ein prädiktiver Marker für ein Therapieansprechen sein, jedoch sollte auch PD-L1-

\section{„Die Studienergebnisse zeigen} einen echten Durchbruch und sind "practice changing".

negativen Patienten die Therapie mit einem PD-1-Inhibitor nicht vorenthalten werden. Die Bedeutung der PD-L1-Positivität, die Wahl der Cut-Offs und die Wertigkeit der verschiedenen Testsysteme werden Gegenstand weiterer Untersuchungen und Ringversuche sein.

Darüber hinaus zeigte sich die Überlegenheit von Pembrolizumab über Ipilimumab unabhängig vom BRAF-Mutationsstatus und unabhängig von einer etwaigen vorausgegangenen BRAF-Inhibitor-Therapie. Pembrolizumab erwies sich als deutlich verträglicher als Ipilimumab, trotz der kontinuierlichen Verabreichung. Insbesondere die unter Ipilimumab gefürchtete autoimmunbedingte Kolitis (Schweregrade 3/4) trat unter Pembrolizumab mit 1,4 bzw. 2,5\% deutlich seltener auf als unter Ipilimumab (7,0\%). Hypo- und Hyperthyreose kamen zwar deutlich häufiger vor, jedoch nur in geringen Schweregraden. Ebenso traten Fatigue, Schwäche und Arthralgien (in geringen Schweregraden) unter Pembrolizumab gehäuft auf. Dies macht deutlich, dass neben einem engmaschigen Nebenwirkungs-Monitoring die psychologische Betreuung der Patienten im Verlauf der Therapie ebenso einen hohen Stellenwert haben sollte, auch um die Therapietreue der Patienten zu erhalten.

Insgesamt sind die Ergebnisse der KEYNOTE-006 gut mit den Ergebnissen der Studie Checkmate-067 [Larkin J et al. N Engl J Med. 2015;373(1):23-34] vergleichbar, auch hier zeigte sich die deutliche Überlegenheit des (in Europa im Juni 2015 zugelassenen) PD-1-Inhibitors Nivolumab über den CTLA-
4-Inhibitor Ipilimumab sowohl in Bezug auf die Wirksamkeit als auch Verträglichkeit.

Die Studienergebnisse zeigen einen echten Durchbruch und sind "practice changing". Als Therapiestandard beim fortgeschrittenen Melanom lösen die PD-1-Inhibitoren Ipilimumab ab. Ermöglicht wird dies durch die First-Line-Zulassungen in Europa.

Die Studienergebnisse zeigen auch, dass das Melanom ein gutes Ziel für die Immuntherapie ist und mit PD-1 ein geeigneter Angriffspunkt gefunden wurde. Offen sind die Fragen nach dem Langzeitüberleben unter PD-1-Blockade, nach der Kombination der verschiedenen Präparate (auch der zielgerichteten Therapie) und den besten Algorithmen/Sequenzen.

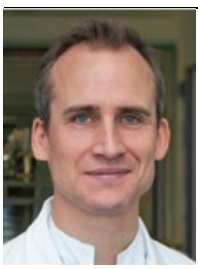

Dr. med. Dirk Debus Hauttumorzentrum Nürnberg Klinikum Nürnberg Nord dirk.debus@klinikumnuernberg.de

\title{
Melanom: Langzeitdaten zur Bestrahlung nach Lymphadenektomie
}

\section{Für Patienten mit Melanom wird} nach einer Lymphadenektomie eine Bestrahlung empfohlen. Nun liegen neue Daten zum Überleben und zu den Nebenwirkungen vor.

$\mathrm{n}$ der randomisierten, kontrollierten Phase-III-Studie ANZMTG 01.02/ TROG 02.01 reduzierte eine Bestrahlung das Risiko für ein Rezidiv in den umliegenden Lymphknoten bei Melanompatienten mit hohem Rezidivrisiko, aber sie hatte keinen Einfluss auf das Gesamtüberleben (OS). Die frühen toxischen Effekte waren akzeptabel niedrig. Nun wurden die Endergebnisse publiziert.

An der Studie nahmen 250 Melanompatienten teil, die ein hohes Rezidivrisiko hatten und sich einer Lymphadenektomie zur Therapie eines palpablen Lymphknotenrezidivs unterzogen. Die Patienten erhielten randomisiert eine adjuvante Bestrahlung (48 Gy in 20 Fraktionen über maximal 30 Tage) oder wurden nur beobachtet. Der primäre End- punkt war ein Lymphknotenrezidiv als erstes Rezidiv. Zudem wurden die späten Toxizitäten registriert, die mehr als 90 Tage nach der Operation oder dem Bestrahlungsbeginn auftraten. Die mediane Nachbeobachtungzeit war 73 Monate.

Rezidive traten bei $21 \%$ der Patienten nach neoadjuvanter Bestrahlung und bei $36 \%$ in der Beobachtungsgruppe auf (adjustierte Hazard Ratio [HR] 0,52; $\mathrm{p}=$ $0,023)$. Das 5-Jahres-OS war in beiden Gruppen vergleichbar (40 vs. $45 \%$; HR $1,27 ; \mathrm{p}=0,21)$, ebenso das rezidivfreie 5-Jahres-Überleben (HR 0,89; $p=0,51$ ). Geringgradige toxische Langzeiteffekte der Bestrahlung, vor allem Schmerzen, Fibrose der Haut (42\%) oder des subkutanen Gewebes (50\%), traten häufig auf. $22 \%$ der Patienten mit adjuvanter Bestrahlung entwickelten toxische Effekte vom Grad 3/4. Zudem stieg das Volumen der unteren Extremitäten nach der adjuvanten Strahlentherapie im Vergleich zur Beobachtungsgruppe signifikant an (mittleres Volumenverhältnis 15,0 vs.
$7,7 \% ; p=0,014)$. Bei Ödemen der oberen Extremitäten gab es keinen signifikanten Unterschied zwischen den Gruppen.

Fazit: Diese Langzeitdaten bestätigen die bisherigen Beobachtungen. Somit könnte die adjuvante Bestrahlung für Patienten in Betracht gezogen werden, bei denen die Krankheitskontrolle im Bereich der umliegenden Lymphknoten ein wichtiger Aspekt ist. Allerdings wurde die Studie durchgeführt, als zielgerichtete Therapien und Immuncheckpointinhibitoren in dieser Indikation noch nicht etabliert waren. Heutzutage sind diese neueren Therapien womöglich die bevorzugte Option für diese $\mathrm{Pa}$ tienten.

Judith Neumaier

Henderson MA et al. Adjuvant lymph-node field radiotherapy versus observation only in patients with melanoma at high risk of further lymph-node field relapse after lymphadenectomy (ANZMTG 01.02/TROG 02.01): 6-year followup of a phase 3 , randomised controlled trial. Lancet Oncol. 2015;16(9):1049-60. 